Research Paper

\title{
Thyrospheres from B-CPAP Cell Line with BRAF and TERT Promoter Mutations have Different Functional and Molecular Features than Parental Cells
}

\author{
Paola Caria ${ }^{1}$, Rita Pillai ${ }^{1}$, Tinuccia Dettori ${ }^{1}$, Daniela Virginia Frau ${ }^{1}$, Patrizia Zavattari ${ }^{1}$, Gabriele Riva ${ }^{2}$, \\ Gabriele Romano², Fabiana Pani ${ }^{3}$, Angela Bentivegna², Roberto Giovannoni², Fabio Pagni², Valeria Sogos ${ }^{1}$, \\ Roberta Vanni ${ }^{1 凶}$ \\ 1. Department of Biomedical Sciences, University of Cagliari, Italy; \\ 2. School of Medicine and Surgery, University of Milano-Bicocca, Italy; \\ 3. Department of Medical Sciences, University of Cagliari, Italy. \\ $\square$ Corresponding author: Roberta Vanni, Department of Biomedical Sciences, University of Cagliari, Cittadella Universitaria 09042 Monserrato (CA) - Italy Tel: \\ 39.070.675.4123; Fax: 39.070.675.4100 vanni@unica.it \\ (c) Ivyspring International Publisher. This is an open access article distributed under the terms of the Creative Commons Attribution (CC BY-NC) license \\ (https://creativecommons.org/licenses/by-nc/4.0/). See http://ivyspring.com/terms for full terms and conditions.
}

Received: 2016.12.21; Accepted: 2017.03.29; Published: 2017.06.03

\begin{abstract}
Human thyroid cancer derived cell lines are widely used to study the mechanisms involved in thyroid carcinogenesis. However, there is limited availability of non-cross-contaminated cancer cell lines derived from papillary thyroid carcinoma (PTC), and the B-CPAP cell line is one of the few such lines. B-CPAP cells have been genetically and cytogenetically well-characterized, but details of their stemness features remain uncertain. Considering that this cell line is extensively used for in vitro studies on thyroid tumorigenesis, we broaden its functional and molecular profiles as well as the tumorigenic capacity. We used functional assays (sphere-forming capacity and efficiency), assessed self-renewal and propagation efficiency and tested in vivo tumorigenicity in Hsd:Athymic Nude-Foxn Inu mice. Expression of markers of stemness, differentiation, and epithelial-mesenchymal transition were estimated at RNA and protein levels in adherent parental cells and sphere-forming cells. Functional aspects and stemness features were compared with normal thyrocytes. Protein expression of xenograft tumors was evaluated by immunohistochemistry. B-CPAP sphere-forming cells were able to form thyrospheres theoretically indefinitely in an appropriate serum-free medium, reverting to the adherent parental cell phenotype when cultured in differentiation medium. Different expression of ALDH1-A1 and CD44 stemness markers and TTF-1 and CK19 differentiation markers allowed discrimination between isolated sphere-forming cells and adherent parental cells, indicating that sphere-forming cells retained stem-like features. In keeping with these observations, tumorigenicity assays confirmed that, relative to parental adherent cells, thyrospheres had enhanced capacity to initiate xenograft tumors. Thyrospheres from normal cell line retained very low functional capacity, as well as different stemness markers expression compared to tumor thyrospheres. Our findings may constitute a useful background to develop an in vitro model for assessing the origin and progression of papillary thyroid carcinoma bearing BRAFV600E and TERT promoter mutations.
\end{abstract}

Key words: thyrosphere, B-CPAP cell line, TERT mutation, papillary thyroid carcinoma.

\section{Introduction}

According to the cancer stem cell hypothesis, tumors are composed of a heterogeneous mixture of cells with distinct proliferative and differentiation capacities [1]. It is thought that only a small subset of these cells, termed cancer stem cells (CSCs), is able to generate new tumors in recipient animals [2]. CSCs possess the capacity of self-renewal, and are responsible for maintaining malignant potential, may acquire migration capacity, and confer resistance to therapy. Several combinations of specific markers and different assays have been used for identifying and isolating CSCs from tumors and tumor cell lines including expression of CD133 and CD44 cell surface markers, the ability to efflux Hoechst 33342 dye, and 
ALDEFLUOR assay [3]. Functional approaches, such as evaluation of the capacity to form non-adherent spheres, are also used. Indeed, most CSCs show the ability to propagate nearly indefinitely in vitro without altering their stemness properties [4]. This feature allows for in vitro enrichment of the isolated initial putative stem population $[5,6]$. Recently, attention has focused on the possible connection between CSCs and the epithelial-mesenchymaltransition (EMT) process. As epithelial cells undergoing EMT, CSCs downregulate epithelial differentiation markers, such as E-cadherin, which are responsible for the loss of cell-cell adhesion capacity, a pivotal change in the progression of many tumors [7]. The existence of CSCs in papillary thyroid carcinoma (PTC), the most common endocrine malignancy, was postulated approximately ten years ago [8]. However, their presence has been demonstrated only recently [9-12]. The initial lack of information on CSCs in such a common tumor (PTC accounts for approximately $80 \%$ of differentiated thyroid carcinoma) [13] is possibly due to difficulties in obtaining suitable surgical material and lack of suitable thyroid-specific enrichment markers capable of discriminating stem-like cells within the heterogeneous tumor mass. The PTC-derived cell line B-CPAP [14, 15] which shares biological features with the primary tumor from which it derives, is one of the few PTC-derived cell lines that is non cross-contaminated with other cell lines [16]. Because the genetic profile of B-CPAP is considered representative of most aggressive forms of PTC, it is extensively used for studying PTC carcinogenesis [17-20].

Among features that have been described are genetic alterations, such as BRAFV600E and TP53 mutations [21], the chromosome profile [14] including the molecular karyotype [22-24], a certain degree of chromosome instability [25], as well as features of epithelial-mesenchymal transition [26]. Nevertheless, functional and molecular studies of putative CSCs have not been extensively reported, as well as the TERT promoter status. Here, we further refined the genotype and broadly dissected the functional and molecular profiles and tumorigenic capacity of B-CPAP cell line.

\section{Material and Methods}

\section{Cell lines, sphere culture, and functional assays}

The human B-CPAP papillary thyroid carcinoma-derived cell line (kindly provided by Prof. Fusco, the University Federico II, Naples, Italy) was grown as a monolayer in culture in Dulbecco's Modified Eagle's Medium/Ham's F-12 (DMEM/F12) supplemented with $10 \%$ fetal bovine serum (FBS, Life
Technologies, Milan, Italy), 2mM L-glutamine and 100 $\mathrm{UI} / \mathrm{ml}$ penicillin and $100 \mu \mathrm{g} / \mathrm{ml}$ streptomycin (Sigma-Aldrich, Milan, Italy), at $37^{\circ} \mathrm{C}$ in a humidified $5 \% \mathrm{CO}_{2}$ atmosphere.

A sphere-forming assay was used to assess self-renewal and sphere-forming efficiency. For generating thyrospheres, B-CPAP monolayer cells were grown for one week with reduced FBS (5\%). Adherent cells were then gently dissociated with StemPro Accutase (Life Technologies), and single cells were cultured in permissive condition, at a density of $2 \times 10^{4}$ cells $/ \mathrm{ml}$ in low-attachment flasks (Corning, Corning, NY, USA), in serum-free medium (SFM) as follows: DMEM/F12 with 2\% B27 supplement (Life Technologies) and epidermal growth factor (EGF), and basic fibroblastic growth factor (bFGF) (Miltenyi Biotec, Calderara di Reno, BO, Italy) $(20 \mathrm{ng} / \mathrm{ml}$ each). Monitoring of thyrosphere formation was done with an Olympus IMT-2 inverted microscope (San Diego, CA, USA); images were captured with a Moticam Pro digital camera (Motic, Seneco, MI, Italy).

To confirm self-renewal, primary thyrospheres of approximately $200 \mu \mathrm{m}$ in size were enzymatically dissociated with StemPro Accutase (Life Technologies) and replated in SFM every seven days at a density of $2 \times 10^{4}$ cells $/ \mathrm{ml}$ to obtain next generation spheres.

To evaluate sphere forming efficiency (SFE), thyrospheres were dissociated and seeded at a cell density of 500 cells/ml in low-attachment 24-well plates (Corning) in SFM. Every 15 days, spheres that had formed were counted, dissociated, and replated to generate new spheres. The numbers of spheres counted were expressed as a percent of total plated cells.

To induce differentiation of thyrospheres into adherent cells, thyrospheres were cultured in medium supplemented with $10 \%$ FBS for at least 4 weeks, and then harvested for molecular analysis.

Functional parameters and stemness markers of $\mathrm{B}-\mathrm{CPAP}$ thyrospheres were compared to thyrospheres isolated (see above) from a normal thyrocytes-derived cell line. Nthy-ori 3-1 (SV-40 immortalized normal human thyroid follicular cells) was purchased from Health Protection Agency Culture Collections. Expression of B-CPAP differentiation markers were in preference compared to normal thyroid tissue.

\section{Reverse Transcription Polymerase Chain Reaction}

Total RNA was extracted from multiple populations including parental cells, various thyrosphere generations, and sphere-derived adherent cells. TRI Reagent Isolation Solution (Sigma-Aldrich) was used according to the 
manufacturer's instructions, and concentration, integrity, and purity were determined by NanoPhotometer ${ }^{\mathrm{TM}} \mathrm{P} 300$ Version 1.0 (Implen GmbH, München, Germany) for each cell subset.

RNA $(1 \mu \mathrm{g})$ was reverse transcribed into cDNA using Oligo (dT) 12-18 primers (Life Technologies) and Moloney Murine Leukemia Virus reverse transcriptase (M-MLV; 200 U; Life Technologies) according to the manufacturer's instructions. cDNA was subsequently amplified by polymerase chain reaction (PCR) according to Lan [27]. Primers and reaction conditions are summarized in Table S1. PCR products were separated by $2 \%$ agarose gel electrophoresis, stained with SYBR Safe (Life Technologies), and visualized with a UV transilluminator (ECX-F20M, CSL-MicroDOCCompact Gel documentation system, Eppendorf, Hamburg, Germany). Signals corresponding to each gene were analyzed using Quantity One software (Bio-Rad Hercules, CA, USA). mRNA amounts were normalized by comparison to GAPDH mRNA levels.

\section{Immunofluorescence staining}

For immunostaining, cells from dissociated thyrospheres were replated in SFM on poly-D-lysine coated coverslips for 2 hours before fixation. Cells were fixed with cold methanol for 4 minutes at $-20^{\circ} \mathrm{C}$ for all antibodies, except that cells to be stained for ABCG2 were fixed in 4\% paraformaldehyde for 10 minutes at room temperature. Immunostaining was performed as previously described [28] using antibodies listed in Table S2. Alexa-conjugated (Alexa Fluor 488 or 594, Life Technologies) goat anti-mouse or anti-rabbit IgG were used as secondary antibodies. Nuclei were counterstained with 4',6-diamidino-2phenylindole (DAPI).

\section{Western Blot analysis}

Cells were lysed with $2 \%$ sodium dodecyl sulfate at $4^{\circ} \mathrm{C}$. Protein concentration was measured according to the method of Lowry [29]. Western blot was carried out as previously described [28] using antibodies listed in Table S2. Horseradish peroxidase-conjugated anti-mouse or anti-rabbit IgG (both from DAKO, Carpinteria, CA, USA) were used as secondary antibodies.

\section{Gene Mutation assay}

Genomic DNA was isolated from both adherent cells and primary thyrospheres using a QIAmp DNA Mini Kit (Qiagen, Milan, Italy). Quantification of genomic DNA was determined by NanoPhotometer (Implen).

\section{BRAFV600E mutation}

Exon 15 of the BRAF gene was amplified by PCR as previously described [30]. Confirmation of mutation was performed by direct sequencing with an ABI 310 Genetic Analyzer (Applied Biosystems, Milan, Italy) utilizing BigDye ver. 1.1 terminator kit (Applied Biosystems) and specific amplification primers for exon 15 (Life Technologies).

\section{TP53 mutation}

Exons 7, 8, and 9 of TP53 were analyzed using primers as previously described [21]. PCR products were amplified with High-Fidelity Taq polymerase (Platinum ${ }^{\circledR}$ Taq DNA Polymerase High Fidelity, Invitrogen), purified (by exonuclease 1 and shrimp alkaline phosphatase) and sequenced by fluorescence-based Sanger's direct sequencing on an ABI 3130 XL DNA capillary sequencer (Applied Biosystems).

\section{TERT promoter mutation}

Standard PCR was carried out for genetic sequencing to identify TERT mutation. Briefly, a fragment of TERT promoter was amplified by PCR on genomic DNA using primers previously described [31]. Thirty nanograms of genomic DNA was used in PCR (initial activation step at $95^{\circ} \mathrm{C}$ for $3 \mathrm{~min}$, forty cycles of $95^{\circ} \mathrm{C}$ denaturation for $30 \mathrm{sec}, 62^{\circ} \mathrm{C}$ annealing for $1 \mathrm{~min}, 72^{\circ} \mathrm{C}$ elongation for $2,5 \mathrm{~min}$, and a final elongation step at $72^{\circ} \mathrm{C}$ for $7 \mathrm{~min}$ ). TERT promoter sequences were generated by direct sequencing with an ABI 310 Genetic Analyzer (Applied Biosystems) utilizing BigDye ver. 1.1 terminator kit (Applied Biosystems).

\section{In vivo tumorigenicity assay}

All the experiments involving animals were carried out in accordance with national and European Guidelines for use and care of laboratory animals, according to a protocol approved by the Italian Ministry of Health (268/2012-B). Four to six week-old female Hsd:Athymic Nude-Foxn1 ${ }^{\text {nu }}$ mice were obtained from Envigo Italy and maintained under specific pathogen-free conditions before xenograft injection. Viability of B-CPAP thyrospheres or adherent cells was evaluated using trypan blue dye exclusion assay before being resuspended in 50\% phosphate buffered saline (PBS)-50\% Cultrex (Trevigen, Inc., Gaithersburg, MD, USA) solution. The cell suspension $(200 \mu \mathrm{l})$ was injected subcutaneously into flanks of Hsd:Athymic Nude-Foxn1 1 nu mice (see results). Tumor growth was measured with a caliper once a week for 7 weeks starting from 7 days post injection. Tumor volume was then calculated according to the following formula: $\mathrm{V}\left(\mathrm{mm}^{3}\right)=\left(\right.$ width $^{2}$ $\times$ length) $/ 2$. At the end of the experiment, mice were euthanized under anesthesia and tumors were 
collected for histological and immunohistochemical analyses. Tumorigenicity efficiency was calculated as percentage of number of tumor formation/total of injection sites.

\section{Histopathology and Immunohistochemistry}

Specimens from tumors were fixed in 10\% neutral formalin for 24 hours, and then paraffin embedded. Sections $(2 \mu \mathrm{m})$ were stained with Hematoxylin \& Eosin for morphological evaluation. Specific PTC nuclear features were scanned including clearing, overlapping, grooves, and pseudoinclusions. The architectural pattern (follicular, papillary, solid, sarcomatoid) was evaluated; areas of necrosis and the mitotic index were recorded. Immunohistochemistry (IHC) was performed after heat-mediated antigen retrieval on a Dako autostainer (Glostrup, Denmark) using specific antibodies listed in Table S2.

\section{Statistical analysis}

Results were analyzed using the 2-tailed Student t-test; $P<0.05$ was considered statistically significant. Statistical analysis was carried out with Prism, 5.00 software (GraphPad Software, Inc, La Jolla, CA).

\section{Results}

\section{Functional characterization}

\section{Primary thyrosphere formation}

A subpopulation of adherent B-CPAP parental cells was able to form thyrospheres in SFM. Specifically, cells growing in suspension formed three-dimensional aggregates - spheroid clusters - at day 3. Spheres were visible after five days and reached the maximum size of 150-300 $\mu \mathrm{m}$ after seven days (Figure 1A). The same behavior was observed for Nthy ori 3-1-derived spheres.

\section{Self-renewal}

To assess the presence of a self-renewing population of stem-like cells within thyrospheres, primary thyrospheres were enzymatically dissociated and re-seeded at the initial cell density. These cells were found to form secondary spheres. B-CPAP cells had an extensive capacity for self-renewal, reaching at least 21 generations (Figure 1B). The Nthy ori 3-1 self-renewal capacity was instead limited to four generations (Supplement Figure 1A).

\section{Sphere-Forming Efficiency (SFE)}

B-CPAP adherent cells had an initial SFE of approximately $2 \%$. This value increased with each iteration, reaching a peak value at generation 8 $(18 \% \pm 2.1)$. Although SFE decreased after generation 8 , cells maintained a stable efficiency of $10 \%$ through the end of the experiment (20th generation) (Figure 1C). The Nthy ori 3-1 thyrosphere SFE was initially high. However, it diminished from the second sphere generation, by which cells start to flat adhering to the flask surface and gaining epithelial-like morphology in not permissive medium (Supplement Figure 1B).

\section{Thyrosphere differentiation}

When B-CPAP thyrospheres were cultured in permissive condition, cells from the outermost layer began flattening and attached to the flask surface usually within $24 \mathrm{~h}$ after seeding. By $72 \mathrm{~h}$ they had acquired an epithelial-like morphology and were indistinguishable from the adherent parental cells (Figure 1D). We refer to these cells as adherent sphere-derived cells.

\section{Molecular characterization}

The B-CPAP molecular profiles are summarized in Table 1 and are as follows.

\section{Reverse Transcription-PCR (RT-PCR)}

The expression of stem cell markers (Oct4, Nanog, ABCG2, p63, and ALDH1-A1) and thyroid differentiation markers (Tg, PAX8, and TTF-1) were evaluated by RT-PCR, and were compared among parental adherent cells, thyrospheres, and adherent sphere-derived cells to determine the effect of SFM on expression of stemness/differentiation genes.

Table 1. Molecular profile of B-CPAP cell subsets

\begin{tabular}{|c|c|c|c|}
\hline & adhP & thys & adhSD \\
\hline \multicolumn{4}{|c|}{ Stemness markers } \\
\hline Oct4 & Pos & pos & pos \\
\hline Nanog & Pos & pos & pos \\
\hline$A B C G 2$ & Pos & pos & pos \\
\hline$A L D H 1-A 1$ & Neg & pos & neg \\
\hline CD44 & Pos & $\operatorname{pos}^{*}$ & pos \\
\hline$p 63$ & Pos & pos & pos \\
\hline \multicolumn{4}{|c|}{ Differentiation markers } \\
\hline TG & Neg & neg & neg \\
\hline TTF-1 & Pos & neg & pos \\
\hline PAX8 & Pos & pos & pos \\
\hline CK19 & Pos & neg & pos \\
\hline \multicolumn{4}{|c|}{ EMT markers } \\
\hline E-cadh & Neg & neg & neg \\
\hline Vimen & Pos & pos & pos \\
\hline \multicolumn{4}{|c|}{$\begin{array}{l}\text { Specific thyroid somatic } \\
\text { mutations }\end{array}$} \\
\hline BRAFV600E & Pos & pos & Pos \\
\hline p53 & Pos & pos & Pos \\
\hline TERT & Pos & pos & Pos \\
\hline
\end{tabular}


A
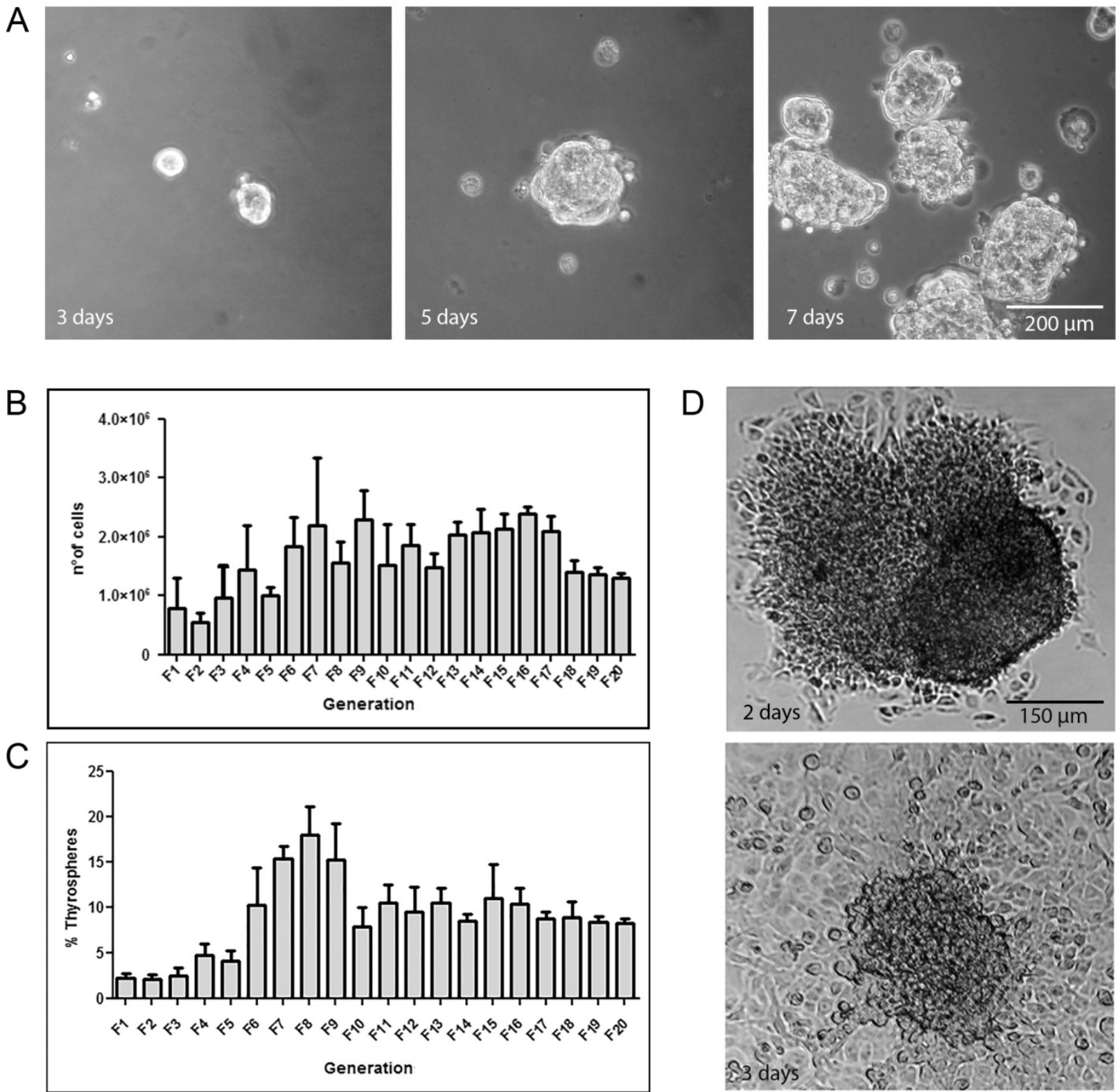

Figure 1. A) B-CPAP thyrosphere morphology during growth in SFM. Morphology at 3, 5, 7 days. B) Self-renewal assay illustrating propagation of B-CPAP-derived thyrospheres. The graph shows the total number of cells ( $Y$ axis) for each generation after 7 days of culture in SFM (X axis). C) Sphere Forming Efficiency showing the maintenance of a stable capacity to generate spheres after generation 10. Data represent the means \pm SD of three independent experiments. D) Thyrosphere in differentiation medium showing a trend toward attaching to the flask at day 2 , and toward acquiring an epithelial-like morphology identical to adherent parental cells at day 3.

Oct4, Nanog, ABCG2, and p63 mRNAs were expressed at comparable levels in all cell subsets; ALDH1-A1 mRNA was expressed in thyrospheres, although it was significantly reduced in adherent parental $(p=0.0020)$ and sphere-derived $(p=0.0016)$ cells (Figure 2A).

Thyroglobulin ( $\mathrm{Tg}$ ) expression was negative; PAX8 was equally expressed in all subsets, and TTF-1 exhibited lower expression in thyrospheres compared to adherent parental cells $(p=0.0109)$ and adherent sphere-derived cells $(\mathrm{p}=0.0056)$ (Figure 2B).

No significant difference in mRNA expression through thyrosphere generations was observed; therefore, immunofluorescence and Western-blot analyses were performed on first generation thyrospheres.

All stemness markers but p63 and ALDH1-A1 were expressed in Nthy ori 3-1 thyrospheres, confirming that ALDH1-A1 was specific of B-CPAP thyrospheres (Supplement Figure 2A).

Normal thyroid tissue expressed TTF1, PAX8 and $\mathrm{Tg}$ (Supplement Figure 2B).

\section{Immunofluorescence}

Thyrospheres were positive for the stem cell marker ALDH1-A1, whereas, TTF-1 differentiation 
marker was not detectable. In contrast, both adherent parental and adherent sphere-derived cells were clearly labeled for TTF-1, although neither showed immunoreactivity for ALDH1-A1. The other tested stem-related markers CD44, ABCG2, and Oct4 were expressed by all cell populations. The expression of two EMT markers, E-cadherin and vimentin, was evaluated in the different cell populations. All analyzed cell subsets were negative for E-cadherin but were positive for vimentin expression, which was more pronounced in thyrospheres (Figures 3A-F).

\section{Western Blot}

Immunoblot analysis confirmed results obtained by RT-PCR and immunofluorescence. ALDH1-A1 protein was expressed exclusively in thyrospheres, whereas, CD44 was present in all cell subsets, although higher expression was observed in thyrospheres compared to adherent parental $(p=0.007)$ and adherent sphere-derived $(p=0.0246)$ cells. p63 was expressed in all cell subsets. In particular, we observed only a $70 \mathrm{kDa}$ band, corresponding to the $\Delta$ Np63alpha isoform (Figure 4A). TTF-1 transcription factor and the differentiation marker CK19 were detected only in adherent parental and adherent sphere-derived cells, thyroglobulin was absent in all cell populations, whereas normal thyroid tissue expressed TTF1, CK19 and Tg (Figure 4B).

Moreover, all B-CPAP cell populations were negative for E-cadherin. Vimentin expression was higher in thyrospheres compared to adherent cell populations although the difference was not statistically significant (Figure 4C).

TERT promoter, BRAF, and TP53 mutations. Both B-CPAP adherent cells and thyrospheres bore TERT C228T, BRAFV600E, and TP53 mutations.

\section{Tumorigenicity}

The ability of B-CPAP thyrospheres to induce tumor formation in immunocompromised mice was

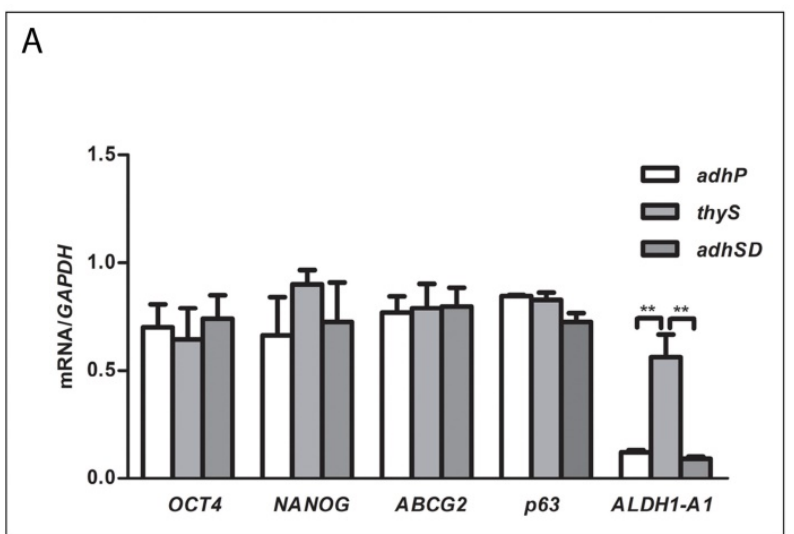

compared to that of the corresponding adherent cells. Thyrosphere cells were more efficient in inducing tumors when subcutaneously xenografted in Hsd:Athymic Nude-Foxn1 ${ }^{\text {nu }}$ mice as compared to adherent cells. When thyrosphere cells and adherent cells were inoculated at the same concentration $\left(5 \times 10^{5}\right.$ cells in $200 \mu \mathrm{l}$ of volume injection) tumor formation was observed in 4 of $6(66.67 \%)$ mice compared to 3 of $8(37.50 \%)$ mice, respectively. Adherent cells remained less efficient ( 3 tumors out of 8 injections) when they were inoculated at increased concentration ( $2 \times 10^{6}$ cells in $200 \mu \mathrm{l}$ of volume injection) (Figure 5A and $\mathrm{B})$, suggesting that B-CPAP thyrospheres were more prone to establish tumors in vivo as compared to B-CPAP parental cells. No significant differences in volume were found between adherent cell-derived and thyrosphere cell-derived tumors over seven weeks.

Morphological analysis of tumor masses of tumors derived from B-CPAP adherent parental cells did not exhibit classic PTC-like nuclear features. Tumors were composed of clusters of cells arranged in solid areas, without specific architectural patterns such as follicular or papillary structures. Necrosis was present. A high power field (HPF) evidenced nuclei with severe atypia, hyperchromasia, nuclear membrane irregularities, and pleomorphism. Frequent mitoses were easily found (2-3x10 HPF). Tumors derived from thyrospheres showed similar features, being composed of solid neoplasms with clear-cut malignant features such as angioinvasion. Coagulative necrosis and focal sarcomatoid-storiform areas were evident. IHC analysis indicated the absence of well-differentiated thyroid tumors markers like TTF-1 and Thyroglobulin. Moreover, CK19, HBME1, and p63 (anti-p-40) were negative. Focal reactivity for Galectin-3 was observed, and tumors exhibited a high proliferative index (Mib-1 30-40\%) (Figure 5C).

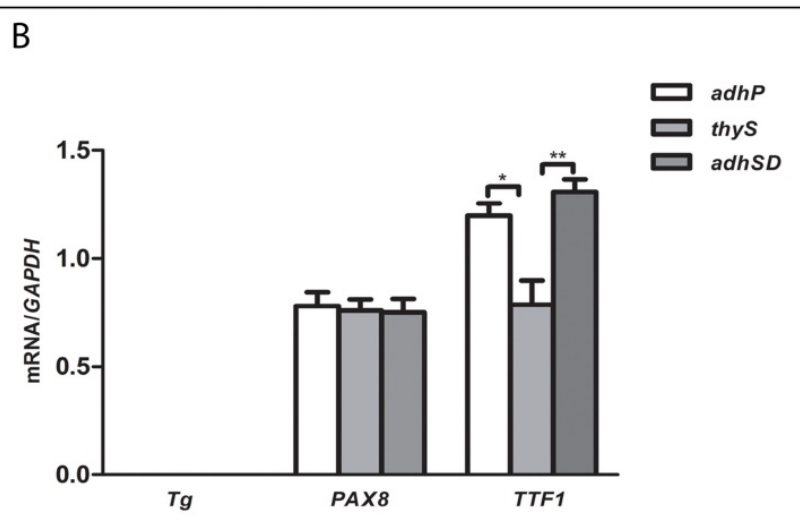

Figure 2. A) Expression levels of Oct-4, Nanog, ABCG2 and ALDH1-A1 mRNA in B-CPAP. B) Expression levels of Tg, PAX8, and TTF-1 mRNA in B-CPAP. Data represent the means $\pm \mathrm{SD}$ of three independent experiments. 

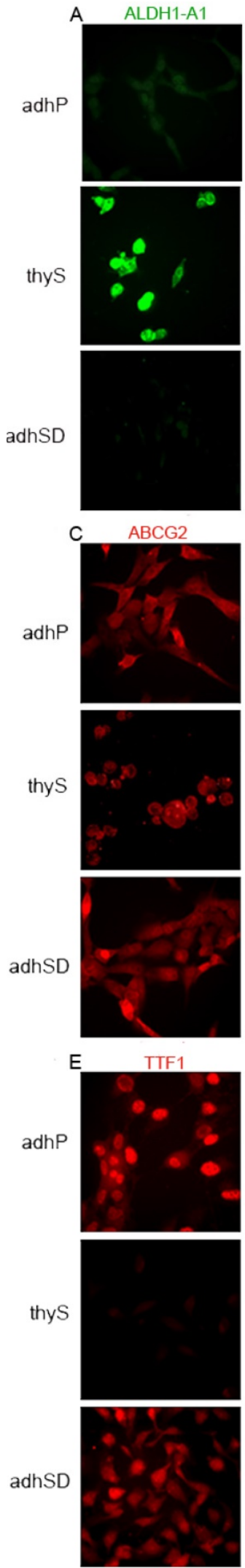
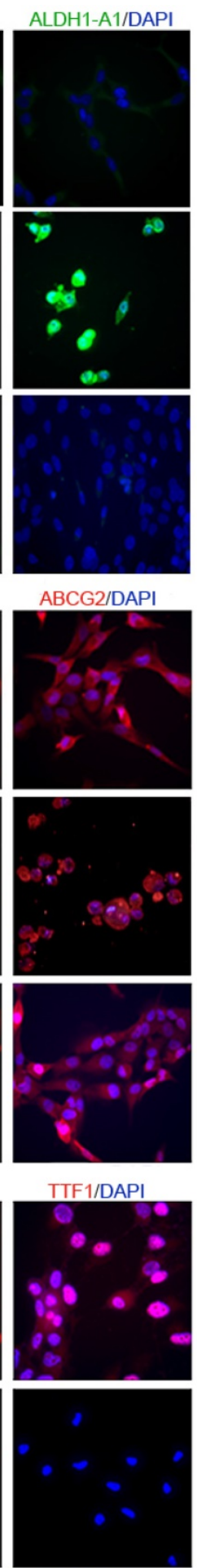

ABCG2/DAPI
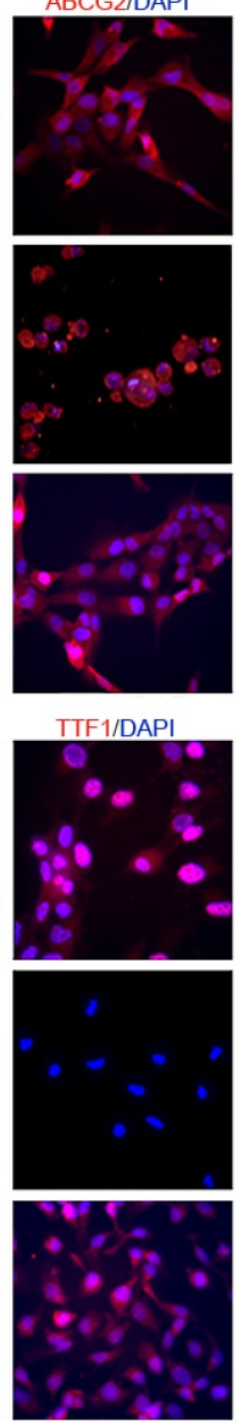

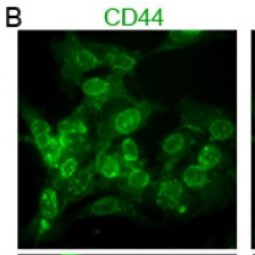

CD44/DAPI
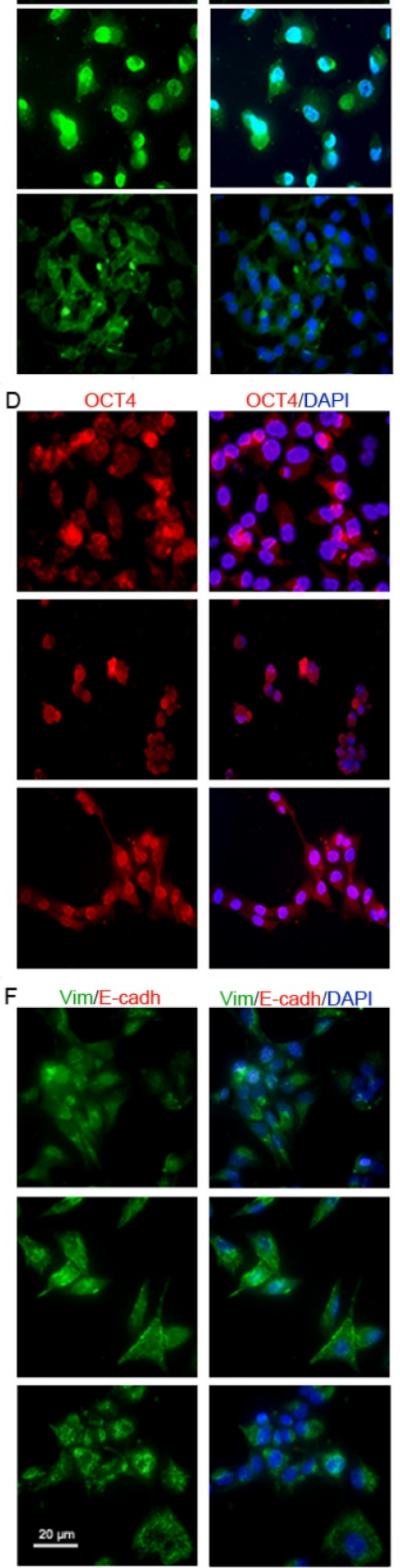

Figure 3. Immunofluorescence pattern in B-CPAP. A) ALDH1-Al (green) positive thyrospheres and negative adherent cells B) CD44 (green) positive cells in all analyzed subsets, with thyrosphere cells showing brighter immunostaining. C) ABCG2 (red) expression in analyzed subsets. D) Oct4 (red) expression in analyzed cell subsets. E) TTF-1 (red) expression in analyzed subsets. F) Vimentin (positive, green)/E-cadherin(negative) double staining in analyzed cell subsets. Nuclei are stained with DAPI (blue). Scale bar: $20 \mu \mathrm{m}$. Images are representative of three independent experiments. Adherent parental cells: adhP; Sphere-Derived cells: adhSD; thyrospheres: thyS. ${ }^{*} \mathrm{p}<0.05 ; * * \mathrm{p}<0.01$. 

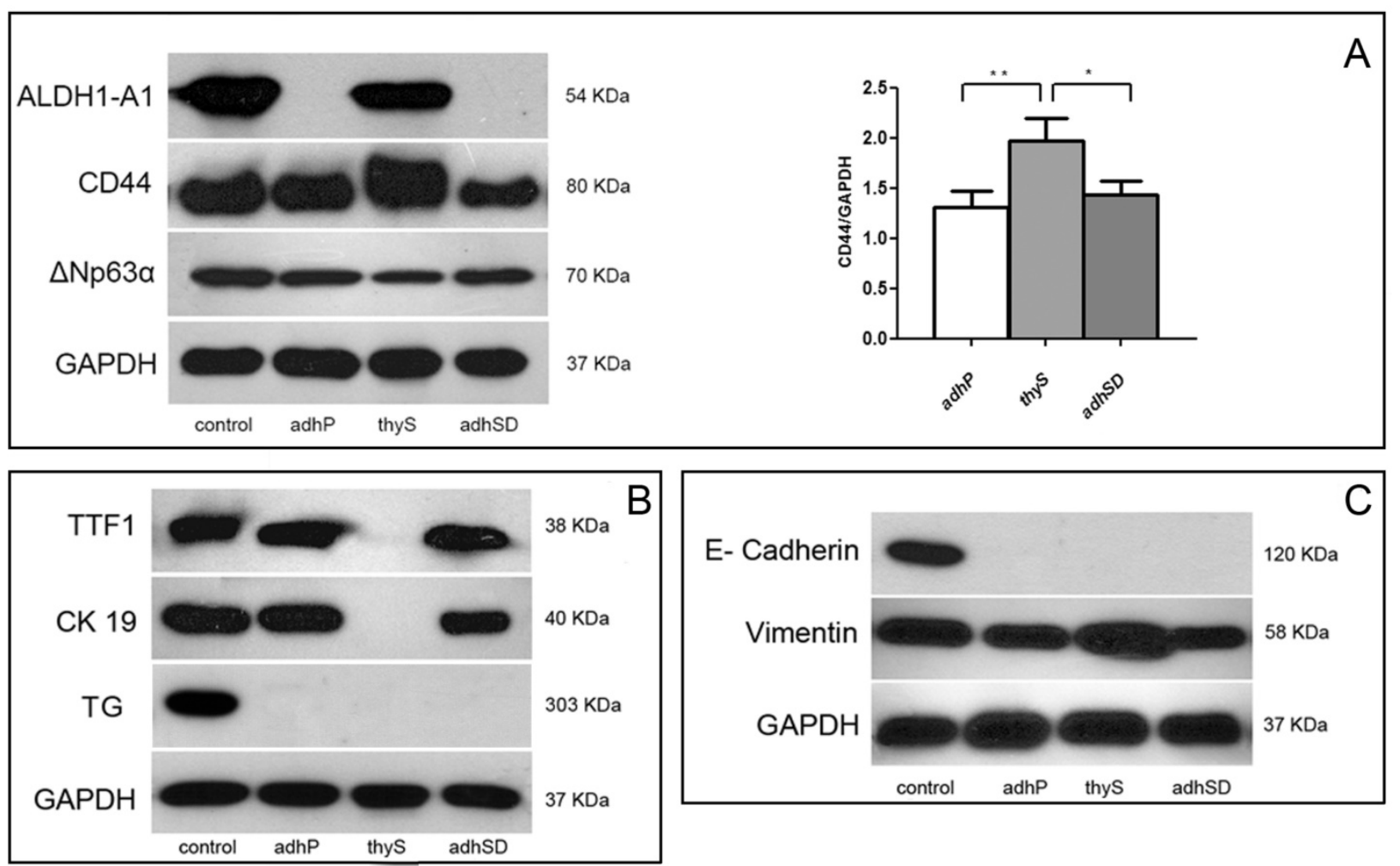

Figure 4. B-CPAP Western blot analysis A) ALDHI-AI, CD44, and $\triangle$ NP63alpha expression in adherent parental cells, adherent Sphere-Derived cells, and thyrospheres (left). Histogram showing expression relative to GAPDH (right). (Data represent the means \pm SD of three independent experiments). B) Expression of $\mathrm{Tg}$, TTF-1 and CK19. C) Expression of E-cadherin and vimentin. Controls: fetal liver (ALDHI-Al and CD44), fetal brain ( $\triangle \mathrm{Np63alpha);} \mathrm{normal} \mathrm{thyroid} \mathrm{(Tg,} \mathrm{CK19,}$ TTF-1, E-cadherin and GAPDH). Adherent parental cells: adhP; adherent Sphere-Derived cells: adhSD; thyrospheres: thyS. ${ }^{*} p<0.05 ; * * p<0.01$.

\section{Discussion}

Despite the recognition that CSCs are present in differentiated primary thyroid tumors [9-11] the contributions of CSCs to generating PTC are poorly investigated. Among the various possible experimental models, human cancer cell lines are frequently used. However, few non cross-contaminated PTC-derived cell lines are available for in vitro studies [16], and those that exist are not fully characterized. Considering this, we widened the characterization of $\mathrm{B}-\mathrm{CPAP}$, one of the most used PTC-derived cell lines, by refining the genetic profile, defining functional aspects and identifying specific biomarkers that allow discrimination between isolated sphere-forming cells (thyrospheres) and parental adherent cells. Moreover, we compared tumorigenicity of the two populations. We isolated cells with thyrosphere-forming capacity from adherent parental cells, and demonstrated that B-CPAP cells have a higher proliferative potential, culminating in the acquisition of putative indefinite self-renewal capacity, then normal thyrocytes. This difference is in agreement with recent data comparing functional aspects of thyrospheres from normal and tumor thyroid tissue [32, 33]. In our hands, stimulation with EGF and bFGF molecules for several generations was indeed needed for B-CPAP to reach maximal self-renewal capacity, sustaining long-term proliferation and stable growth. Mutated TP53, which characterizes B-CPAP cells, might favor this behavior. Cicalese and coworkers [34] have demonstrated that unperturbed levels of p53 impose asymmetrical division in mammary stem cells, whereas, loss of p53 favors symmetrical division. Moreover, a potential role of the mutated form of p53 on the deregulation of the self-renewal/differentiation program of CSCs has been suggested [35]. In addition, although p63 was equally expressed in adherent and thyrosphere cells, we observed that B-CPAP cells showed only the $\triangle$ Np63alpha isoform. $\triangle$ Np63alpha is the main isoform in epithelial tissues and is essential for the self-renewal of adult stratified epithelial [36] and glandular structures [37]. As the expression of p63 in thyroid tumors is controversial, the finding of this specific isoform in B-CPAP cells reinforces the idea that $\triangle$ Np63alpha might regulate self-renewal of $\mathrm{B}-\mathrm{CPAP}$ thyrospheres, and possibly has a role in thyroid carcinoma proliferation in general. Recent data on $\triangle N p 63 a l p h a$ expression in mammary CSCs indicate that $\Delta \mathrm{Np} 63$ alpha promotes self-renewal and expansion [38]. Intriguingly, p63 expression has been detected in thyroid solid cell nests, which may represent a pool of stem cells of the adult thyroid [39]. 
A
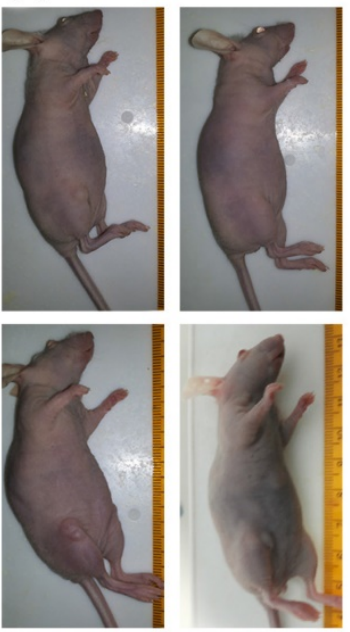

C

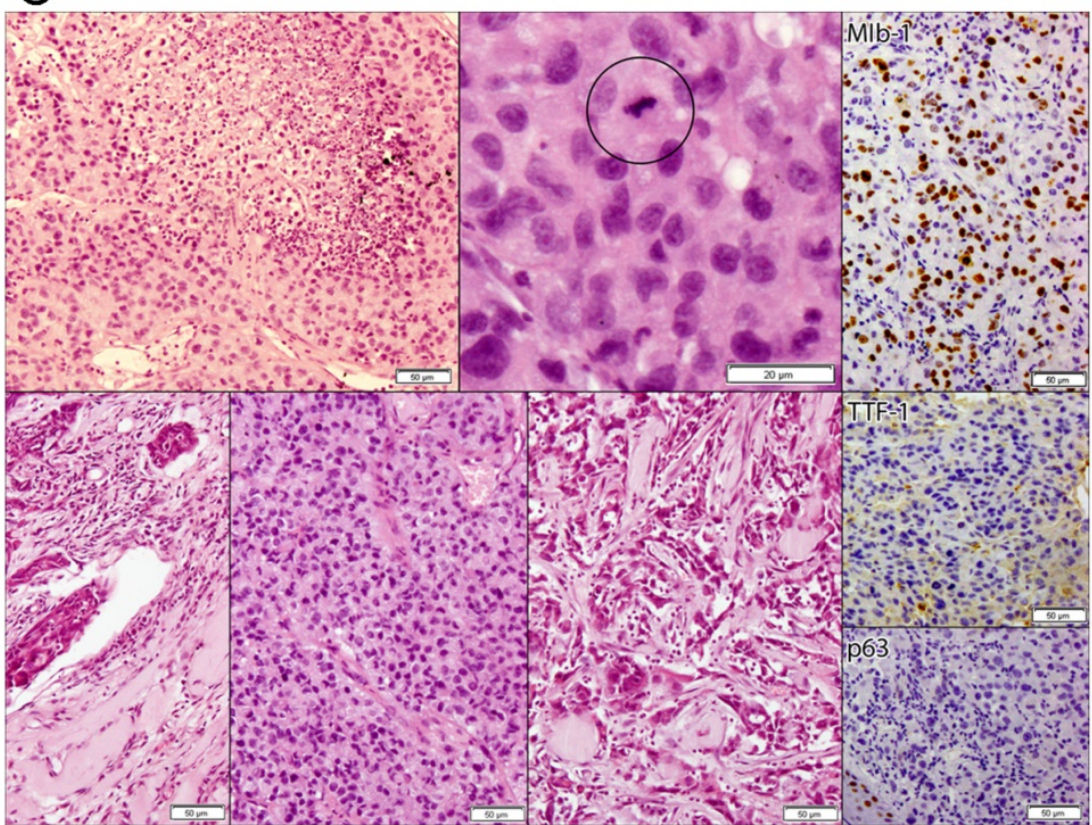

B

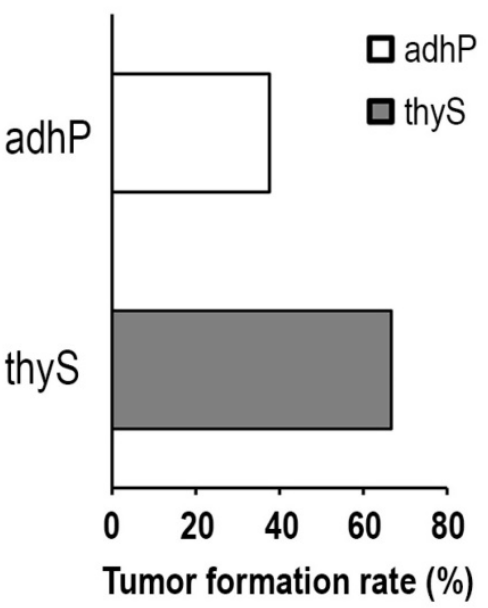

Figure 5. A) Representative images of xenografted B-CPAP grown as adherent cells (upper images) or as thyrosphere (lower images). B) Percentages of tumor masses after 49 days subcutaneous injection of adherent (adhP) or thyrosphere (thyS) B-CPAP cells. C) Representative pictures of tumors from xenografted B-CPAP grown as adherent cells (upper Hematoxylin-Eosin stained images) or as thyrosphere (lower H\&E stained images). Morphological analysis of tumors from adherent cells revealed a high-grade thyroid carcinoma with spread coagulative necrosis (upper left, H\&E 20X). Frequent mitoses were present (black circle, 40X). Histology in thyrospheres showed diagnostic features of anaplastic-like carcinoma, too. Angioinvasion was evident (inner left, H\&E, 20X). Tumor architecture was solid and sarcomatoid (H\&E 20X). Immunohistochemical staining for Mibl confirmed a high proliferative index (around 30\%; 20X), TTF-1 (20X), and squamous marker p63 were negative (20X; positive internal control in mouse skin).

We hypothesize that TP53 mutation and $\Delta$ Np63alpha expression might contribute to promoting B-CPAP self-renewal. Among the other tested stemness-related markers, the expression of ALDH1-A1 distinguished putative CSCs from adherent parental cells. ALDH1-A1 is a major ALDH family enzyme involved in the oxidation of retinol to retinoic acid in early stem cell differentiation [40]. Recently, its role as a CSC marker has been reported, as well as its association with poor prognosis in many cancers including lung [41], breast [42], prostate [43], thyroid [9, 44], head and neck [45], and renal [46] 
addition, B-CPAP thyrospheres and parental cells harbored BRAFV600E mutation. In line with the aggressiveness of the primary tumor from which $\mathrm{B}-\mathrm{CPAP}$ has been derived, a synergistic effect of TERT promoter mutation in combination with BRAFV600E on poor clinicopathologic outcomes of PTC has been recently proposed [50]. Accordingly, we also observed higher expression of CD44 stem marker in thyrospheres than parental cells, lack of expression of TTF-1 and CK19 differentiation markers, downregulation of E-cadherin epithelial marker, and upregulation of vimentin mesenchymal marker. In aggregate, these findings suggested that sphere-forming cells had tumorigenic potential, which was confirmed in vivo using immunocompromised mice. Intriguingly, it has been reported that parental B-CPAP cells establish heterotopic tumors in immunocompromised mice (15) at concentrations 6 fold and 1,5 fold higher than the ones we used for testing tumorigenicity of B-CPAP thyrospheres and parental cells, respectively. This suggests that our thyrospheres were enriched for tumor-initiating cells by at least 4 folds compared to parental cells. The IHC analysis of tumor masses confirmed a high proliferation index and absence of classic PTC-markers such as CK19, TTF-1, and HBME1. Lack of these markers is a feature of CSC-rich anaplastic thyroid carcinoma [51], and it is also in line with enrichment of stem-like cancer cells in thyrospheres. As a whole, the profile of this cell line is consistent with that of cancer stem-like cell populations observed in aggressive papillary thyroid carcinoma $[32,33,52]$.

\section{Conclusions}

The B-CPAP cell line, characterized by TERT promoter mutation, BRAFV600E, and TP53 mutations, possess a subpopulation of cells with cancer stem-like properties and is capable of forming thyrospheres. Thyrosphere cells have a putative indefinite self-renewal capacity. Compared to adherent cells they are characterized by differential expression of two stemness markers (ALDH1-A1 and CD44) and two differentiation markers (TTF-1 and CK19) and have higher tumorigenic potential. The characterization of sphere-forming cells from the $\mathrm{B}-\mathrm{CPAP}$ cell line reported here may contribute to the development of a useful in vitro model for dissecting the origin and progression of papillary thyroid carcinoma.

\section{Abbreviations}

bFGF: basic fibroblastic growth factor; CSCs: cancer stem cells; DAPI: 4',6-diamidino-2phenylindole; DMEM/F12: Dulbecco's Modified
Eagle's Medium/Ham's F-12; EGF: epidermal growth factor; EMT: epithelial-mesenchymal-transition; FBS: fetal bovine serum; H\&E: hematoxylin-eosin; HPF: high power field; IHC: immunohistochemistry; PBS: phosphate buffered saline; PTC: papillary thyroid carcinoma; RT-PCR: reverse transcription polymerase chain reaction; SFE: sphere forming efficiency; SFM: serum-free medium.

\section{Supplementary Material}

Supplementary figures and tables. http://www.jcancer.org/v08p1629s1.pdf

\section{Acknowledgements}

Authors are grateful to A. Cappai and L. Moi for mutational analysis, and $\mathrm{M}$. Aliquò and S. Ielapi for technical assistance in the histopathological analysis.

\section{Funding}

This work was supported by the program "Projects of national interest (PRIN)" of the Italian Ministry of Education, University and Research (MIUR) grant N. 20122ZF7HE (to R Vanni).

\section{Authors' Contributions}

Authors PC, RP, VS, and RV designed the study and wrote the protocol. Authors PC, RP, DVF, TD, FPani and $\mathrm{PZ}$ performed the laboratory experiments. Authors $A B, R G, G R$, and GR performed the tumorigenicity experiments. Author FPagni performed the histopathological analysis of mouse tumors. All authors contributed to and have approved the final manuscript.

\section{Ethics approval}

All procedures and experiments were carried out under approval of the appropriate authorities according to European Directive (63/2010/EU) and Italian law (D.Lgs. 26/2014) on the use of animals for scientific purposes: protocol approved by Italian Ministry of Health N. 268/2012-B. All possible efforts were made to minimize animal pain and discomfort and to reduce the number of experimental subjects.

\section{Competing Interests}

The authors have declared that no competing interest exists.

\section{References}

1. Islam F, Qiao B, Smith RA, et al. Cancer stem cell: fundamental experimental pathological concepts and updates. Exp Mol Pathol. 2015; 98: 184-91.

2. Heppner GH, Miller BE. Tumor heterogeneity: biological implications and therapeutic consequences. Cancer Metastasis Rev. 1983; 2: 5-23.

3. Akbari-Birgani S, Paranjothy $\mathrm{T}$, Zuse $\mathrm{A}$, et al. Cancer stem cells, cancer-initiating cells and methods for their detection. Drug Discov Today. 2016; 21: 836-42

4. Cao L, Zhou Y, Zhai B, et al. Sphere-forming cell subpopulations with cancer stem cell properties in human hepatoma cell lines. BMC Gastroenterol. 2011; 11: 71. 
5. Ponti D. Isolation and In vitro Propagation of Tumorigenic Breast Cancer Cells with Stem/Progenitor Cell Properties. Cancer Res. 2005; 65: 5506-11.

6. Fujii H, Honoki K, Tsujiuchi T, et al. Sphere-forming stem-like cell populations with drug resistance in human sarcoma cell lines. Int J Oncol. 2009; 34: 1381-6.

7. Mani SA, Guo W, Liao MJ, et al. The epithelial-mesenchymal transition generates cells with properties of stem cells. Cell. 2008; 133: 704-15.

8. Zhang P, Zuo H, Ozaki T, et al. Cancer stem cell hypothesis in thyroid cancer. Pathol Int. 2006; 56: 485-9.

9. Todaro M, Iovino F, Eterno $\mathrm{V}$ et al. Tumorigenic and metastatic activity of human thyroid cancer stem cells. Cancer Res. 2010; 70: 8874-85.

10. Malaguarnera R, Frasca F, Garozzo A, et al. Insulin receptor isoforms and insulin-like growth factor receptor in human follicular cell precursors from papillary thyroid cancer and normal thyroid. J Clin Endocrinol Metab. 2011; 96: 766-74

11. Ahn SH, Henderson YC, Williams MD, et al. Detection of thyroid cancer stem cells in papillary thyroid carcinoma. J Clin Endocrinol Metab. 2014; 99: 536-44.

12. Ma R, Minsky N, Morshed SA, et al. Stemness in human thyroid cancers and derived cell lines: the role of asymmetrically dividing cancer stem cells resistant to chemotherapy. J Clin Endocrinol Metab. 2014; 99: E400-9.

13. Nikiforov YE, Nikiforova MN. Molecular genetics and diagnosis of thyroid cancer. Nat Rev Endocrinol. 2011; 7: 569-80.

14. Paulin C, Fabien N, Fusco A, et al. Description of cell line established from human thyroid papillary cancer and secreting human chorionic gonadotropin hormone. C R Acad Sci III. 1992; 315: 493-8.

15. Fabien N, Fusco A, Santoro M, et al. Description of a human papillary thyroid carcinoma cell line. Morphologic study and expression of tumoral markers. Cancer. 1994; 73: 2206-12

16. Schweppe RE, Klopper JP, Korch C, et al. Deoxyribonucleic acid profiling analysis of 40 human thyroid cancer cell lines reveals cross-contamination resulting in cell line redundancy and misidentification. J Clin Endocrinol Metab. 2008; 93: 4331-41.

17. Coperchini F, Pignatti P, Leporati P, et al. Normal human thyroid cells, BCPAP, and TPC- 1 thyroid tumor cell lines display different profile in both basal and TNF-a-induced CXCL8 secretion. Endocrine. 2016; 54: 123-128.

18. Mo XM, Li L, Zhu P, et al. Up-regulation of Hsp27 by ERa/Sp1 facilitates proliferation and confers resistance to apoptosis in human papillary thyroid cancer cells. Mol Cell Endocrinol. 2016; 431:71-87.

19. Hardin $\mathrm{H}$, Guo Z, Shan $W$, et al. The roles of the epithelial-mesenchymal transition marker PRRX1 and miR-146b-5p in papillary thyroid carcinoma progression. Am J Pathol. 2014; 184: 2342-54.

20. Anania M, Gasparri F, Cetti E, et al. Identification of thyroid tumor cell vulnerabilities through a siRNA-based functional screening. Oncotarget. 2015; 27: $34629-48$

21. Meireles AM, Preto A, Rocha AS, et al. Molecular and genotypic characterization of human thyroid follicular cell carcinoma-derived cell lines. Thyroid. 2007; 17: 707-15

22. Corso C, Ulucan H, Parry EM, et al. Comparative analysis of two thyroid tumor cell lines by fluorescence in situ hybridization and comparative genomic hybridization. Cancer Genet Cytogenet. 2002; 137:108-18 20.

23. Dettori T, Frau DV, Garcia JL, et al. Comprehensive conventional and molecular cytogenetic characterization of B-CPAP, a human papillary thyroid carcinoma-derived cell line. Cancer Genet Cytogenet. 2004; 151: 171-7.

24. Ribeiro FR, Meireles AM, Rocha AS, et al. Conventional and molecular cytogenetics of human non-medullary thyroid carcinoma: characterization of eight cell line models and review of the literature on clinical samples. BMC Cancer. 2008; 8: 371

25. Maric I, Viaggi S, Caria P, et al. Centrosomal and mitotic abnormalities in cell lines derived from papillary thyroid cancer harboring specific gene alterations. Mol Cytogenet. 2011; 4: 26

26. Sancisi V, Gandolfi G, Ragazzi M, et al. Cadherin 6 Is a New RUNX2 Target in TGF- $\beta$ Signalling Pathway. PLoS One. 2013; 8: e75489.

27. Lan L, Cui D, Nowka $K$, et al. Stem cells derived from goiters in adults form spheres in response to intense growth stimulation and require thyrotropin for differentiation into thyrocytes. J Clin Endocrinol Metab. 2007; 92: 3681-8.

28. Pillai R, Scintu F, Scorciapino L, et al. Human astrocytes can be induced to differentiate into cells with neuronal phenotype. Exp Cell Res. 2006; 312: 2336-46

29. Lowry OH, Rosebrough NJ, Farr AL, Randall RJ. Protein measurement with the Folin phenol reagent. J Biol Chem. 1951; 193: 265-75.

30. Caria P, Dettori T, Frau DV, et al. Assessing RET/PTC in thyroid nodule fine-needle aspirates: The FISH point of view. Endocr Relat Cancer. 2013; 20: $527-36$

31. Liu T, Wang N, Cao J, et al. The age- and shorter telomere-dependent TERT promoter mutation in follicular thyroid cell-derived carcinomas. Oncogene. 2014;33: 4978-84

32. Giani F, Vella V, Nicolosi ML, et al. Thyrospheres from normal or malignant thyroid tissue have different biological, functional, and genetic features. J Clin Endocrinol Metab. 2015; 100: E1168-78.

33. Cirello V, Vaira V, Grassi ES, et al. Multicellular spheroids from normal and neoplastic thyroid tissues as a suitable model to test the effects of multikinase inhibitors. Oncotarget. 2017; 8: 9752-9766.

34. Cicalese A, Bonizzi G, Pasi CE, et al. The Tumor Suppressor p53 Regulates Polarity of Self-Renewing Divisions in Mammary Stem Cells. Cell. 2009; 138: 1083-95
35. Ginestier C, Charafe-Jauffret E, Birnbaum D. p53 and cancer stem cells: the mevalonate connexion. Cell Cycle. 2012; 11: 2583-4.

36. Senoo M, Pinto F, Crum CP, et al. p63 Is Essential for the Proliferative Potential of Stem Cells in Stratified Epithelia. Cell. 2007; 129: 523-36.

37. Candi E, Rufini A, Terrinoni A, et al. DeltaNp63 regulates thymic development through enhanced expression of FgfR2 and Jag2. Proc Natl Acad Sci. U. S. A. 2007; 104: 11999-2004

38. Memmi EM, Sanarico AG, Giacobbe A, et al. p63 sustains self-renewal of mammary cancer stem cells through regulation of Sonic Hedgehog signaling. Proc Natl Acad Sci. 2015; 112: 3499-504.

39. Preto A, Cameselle-Teijeiro J, Moldes-Boullosa J, et al. Telomerase expression and proliferative activity suggest a stemcell role for thyroid solid cell nests. M Mod Pathol. 2004; 17: 819-26.

40. Marcato P, Dean C, Giacomantonio C, et al. Aldehyde dehydrogenase: Its role as a cancer stem cell marker comes down to the specific isoform. Cell Cycle. 2011; 10: 1378-84.

41. Jiang F, Qiu Q, Khanna A, et al. Aldehyde dehydrogenase 1 is a tumor stem cell-associated marker in lung cancer. Mol Cancer Res. 2009; 7: 330-8.

42. Charafe-Jauffret E, Ginestier C, Iovino F, et al. Aldehyde dehydrogenase 1-positive cancer stem cells mediate metastasis and poor clinical outcome in inflammatory breast cancer. Clin Cancer Res. 2010; 16: 45-55.

43. Li T, Su Y, Mei Y, Leng Q, et al. ALDH1A1 is a marker for malignant prostate stem cells and predictor of prostate cancer patients' outcome. Lab Invest. 2010; 90: 234-44

44. Xing Y, Luo DY, Long MY, et al. High ALDH1A1 expression correlates with poor survival in papillary thyroid carcinoma. World J Surg Oncol. 2014; 12: 29.

45. Clay MR, Tabor M, Owen JH, et al. Single-marker identification of head and neck squamous cell carcinoma cancer stem cells with aldehyde dehydrogenase. Head Neck. 2010; 32: 1195-201.

46. Ozbek E, Calik G, Otunctemur A, et al. Stem cell markers aldehyde dehydrogenase type 1 and nestin expressions in renal cell cancer. Arch Ital Urol Androl. 2012; 84: 7-11.

47. Liu R, Xing M. TERT promoter mutations in thyroid cancer. Endocr Relat Cancer. 2016; 23: 143-55.

48. Huang FW, Bielski CM, Rinne ML, et al. TERT promoter mutations and monoallelic activation of TERT in cancer. Oncogenesis. 2015; 4: e176.

49. Chiba K, Johnson JZ, Vogan JM et al. Cancer-associated TERT promoter mutations abrogate telomerase silencing. eLife 2015; 4: e07918.

50. Shen X, Liu R, Xing M. A six-genotype genetic prognostic model for papillary thyroid cancer. Endocr Relat Cancer. 2017; 24: 41-52.

51. Yun JY, Kim YA, Choe JY, et al. Expression of cancer stem cell markers is more frequent in anaplastic thyroid carcinoma compared to papillary thyroid carcinoma and is related to adverse clinical outcome. J Clin Pathol. 2014; 67: 125-33.

52. Dima M, Pecce V, Biffoni M, et al. Molecular profiles of cancer stem-like cell populations in aggressive thyroid cancers. Endocrine 2016; 53: 145-56. 